\title{
Main Line Fault Localization Methodology in Smart Grid - Part 1: Extended TM2 Method for the Overhead Medium-Voltage Broadband over Power Lines Networks Case
}

\author{
Athanasios G. Lazaropoulos ${ }^{1}$ \\ 1: School of Electrical and Computer Engineering / National Technical University of Athens / \\ 9 Iroon Polytechniou Street / Zografou, GR 15780
}

Received June 13, 2017; Accepted September 2, 2017; Published September 27, 2017

\begin{abstract}
These three papers cover the overall methodology for the identification and localization of faults that occur in main transmission and distribution lines when broadband over power lines (BPL) networks are deployed across the transmission and distribution power grids, respectively. In fact, this fault case is the only one that cannot be handled by the combined operation of Topology Identification Methodology (TIM) and Instability Identification Methodology (FIIM). After the phase of identification of main distribution line faults, which is presented in this paper, the main line fault localization methodology (MLFLM) is applied in order to localize the faults in overhead medium-voltage BPL (OV MV BPL) networks.

The main contribution of this paper, which is focused on the identification of the main distribution line faults, is the presentation of TM2 method extension through the adoption of coupling reflection coefficients. Extended TM2 method is analyzed in order to identify a main distribution line fault regardless of its nature (i.e., short- or open-circuit termination). The behavior of the extended TM2 method is assessed in terms of the main line fault nature and, then, its results are compared against the respective ones during the normal operation, which are given by the original TM2 method, when different main distribution line fault scenarios occur. Extended TM2 method acts as the introductory phase (fault identification) of MLFLM.
\end{abstract}

Keywords: Smart Grid; Intelligent Energy Systems; Broadband over Power Lines (BPL) Networks; Power Line Communications (PLC); Faults; Fault Analysis; Fault Localization; Distribution Power Grids

\section{Introduction}

During the past few years, a tremendous development in the deployment of broadband over power lines (BPL) networks for enhancing the intelligence, stability and autonomy of the vintage power grid infrastructure has been witnessed [1], [2]. Only considering the scale of transmission and distribution power grids in the countries of modern world, BPL technology can transform these traditional grids into an integrated intelligent IP-based communications network with a myriad of smart grid applications [3]-[5].

Apart from the size of today's power grids, the recent interest in smart grids stems from the significant increase in electricity needs of our societies, the need for a more 
interconnected power grid and a more dynamic manner of power management. Therefore, the demand for coordination, agility and feedback of a new global interconnected power grid implies the delivery of high-bandwidth smart grid applications with data rates that exceed $1 \mathrm{Gbps}$ due to the vast amount of information required across the grid. Since the transmission and distribution power grids were not originally intended for conveying high frequency signals, any communication across the grids would be exposed to severe adversarial factors, such as high and frequency-selective channel attenuation and noise [6]-[12].

As concerns the determination of the channel attenuation and reflection coefficient of overhead medium-voltage (OV MV) BPL networks, the well-established hybrid method, which is employed to examine the behavior of various multiconductor transmission line (MTL) structures, is also adopted in this paper [4], [6]-[10], [13]-[24]. Given as inputs the OV MV BPL network topology, OV MV MTL configuration and the applied coupling scheme, the hybrid method gives as outputs the corresponding transfer function and reflection coefficients. Actually, hybrid method consists of: (i) a bottom-up approach that is based on the MTL theory, eigenvalue decomposition (EVD) and singular value decomposition (SVD); and (ii) a top-down approach that is denoted as TM2 method and is based on the concatenation of multidimensional chain scattering matrices. In this paper, TM2 method of the hybrid method, which is analytically presented in [17], is extended in order to cope with the various load terminations of the main line (terminal loads) of the distribution BPL networks since the original TM2 method assumes that terminal loads of OV MV BPL networks are matched.

Apart from the aforementioned adversarial factors that deteriorate the quality of service of OV MV BPL networks, a number of serious problematic conditions that causes temporary or permanent damage to the integrity of power grid can jeopardize the uninterrupted operation and availability of the power distribution. According to [23]-[27], depending on the affected pieces of power grid equipment, the problematic conditions can be divided into two main categories, say: faults and instabilities. In accordance with [27], the fault category describes all the interruptions that may occur across the main and branch lines of a power grid. Between these two cases, it comprises the fault subcategory of main line fault that defines the main interest of this paper. In fact, the main line fault subcategory forms the only fault case which cannot be treated by Topology Identification Methodology (TIM) and Fault and Instability Identification Methodology (FIIM) in [25], [26]. Since main distribution line faults can be assumed to behave as either short- or open-circuit terminal loads depending on the location of the conductors of the main distribution lines after the fault, the behavior of the extended TM2 method during the determination of its reflection coefficients of the aforementioned terminal loads is first examined in this paper. Furthermore, the reflection coefficients of the extended TM2 method during main distribution line faults are compared against the respective ones of the original TM2 method during the normal operation of OV MV BPL networks. The comparison of the reflection coefficients between the normal and fault condition is going to determine the fault appearance across main distribution lines.

The rest of this paper is organized as follows: In Sec.II, the OV MV MTL configuration, the indicative OV MV BPL topologies, the bottom-up approach of the hybrid method and the main distribution line fault subcategory are presented. Sec.III deals with the top-down approach of the hybrid method and, especially, with the extension of TM2 method. Special attention is given to the determination of the reflection coefficients of OV MV BPL topologies when main distribution line faults occur. 
In Sec.IV, numerical results are provided, aiming at marking out the behavior of the extended TM2 method as well as the reflection coefficient differences between the extended and original TM2 method during the normal and fault operation, respectively. Sec.V recapitulates the conclusions of this paper.

\section{OV MV MTL Configurations, OV MV BPL Topologies, Bottom-Up Approach of the Hybrid Method and Faults 2.1 OV MV MTL Configuration}

The OV MV MTL configuration, which is examined in this paper, is presented in Fig. 1(a) of [4]. The OV MV MTL configuration consists of the three phase lines ( $n^{\mathrm{OVMV}}=3$ ) of radius $r_{\mathrm{MV}, \mathrm{p}}$ that are spaced by $\Delta_{\mathrm{MV}}$ and hung at typical heights $h_{\mathrm{MV}}$ above ground. The imperfect ground is considered as the reference conductor with conductivity $\sigma_{\mathrm{g}}$ and relative permittivity $\varepsilon_{\mathrm{rg}}$. The exact values concerning the aforementioned properties are reported in [6], [7], [16], [18], [20], [28]-[30] while the analysis concerning the impact of imperfect ground on broadband signal propagation and transmission via OV MV MTL configurations are analyzed in [6], [7], [16], [18], [20], [31]-[33].

\subsection{Indicative OV MV BPL Topologies}

To cope with the significant BPL signal aggravation due to the channel attenuation and noise, OV MV BPL networks are divided into cascaded OV MV BPL topologies of average path lengths of the order of $1000 \mathrm{~m}$ which are bounded by BPL repeaters. With reference to Fig. 1(a), a typical OV MV BPL topology is presented that is bounded by two repeaters at the position A and B. Arbitrarily, the repeater at the position A acts as the transmitting end whereas the other repeater acts as the receiving end. Depending on the number and length of the branches encountered across the BPL signal propagation, different OV MV BPL topologies may be considered. In these three papers, four indicative OV MV BPL topologies of average path length are examined, namely:

1. A typical urban topology (denoted as urban case) with $N=3$ branches ( $L_{1}=500 \mathrm{~m}, L_{2}=200 \mathrm{~m}, L_{3}=100 \mathrm{~m}, L_{4}=200 \mathrm{~m}, L_{\mathrm{b} 1}=8 \mathrm{~m}, L_{\mathrm{b} 2}=13 \mathrm{~m}, L_{\mathrm{b} 3}=10 \mathrm{~m}$ ).

2. A typical suburban topology (denoted as suburban case) with $N=2$ branches ( $L_{1}=500 \mathrm{~m}, L_{2}=400 \mathrm{~m}, L_{3}=100 \mathrm{~m}, L_{\mathrm{b} 1}=50 \mathrm{~m}, L_{\mathrm{b} 2}=10 \mathrm{~m}$ ).

3. A typical rural topology (denoted as rural case) with only $N=1$ branch ( $L_{1}=600 \mathrm{~m}, L_{2}=400 \mathrm{~m}, L_{\mathrm{b}}=300 \mathrm{~m}$ ).

4. The "LOS" transmission along the same end-to-end distance $L=L_{1}+\ldots+L_{N+1}=1000 \mathrm{~m}$ (denoted as "LOS" case) when no branches are encountered. This topology corresponds to Line of Sight transmission in wireless channels. 


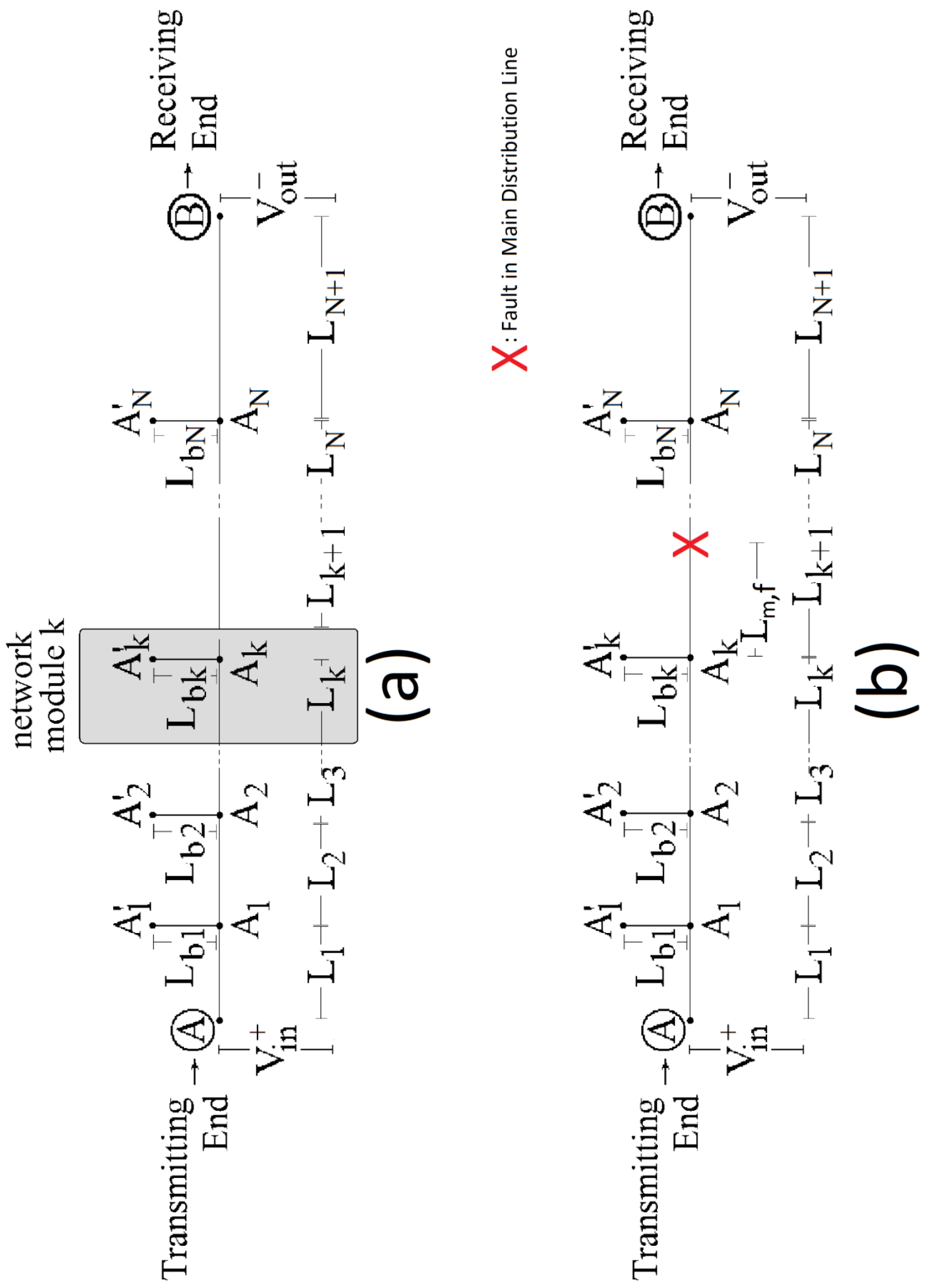

Figure 1. (a) General OV MV BPL topology [23]. (b) Main Distribution Line Fault in OV MV BPL topologies. 


\subsection{Bottom-Up Approach of the Hybrid Method, Coupling Schemes, Coupling} Transfer Functions and Reflection Coefficients

Successfully tested in various transmission and distribution BPL networks [6]-[10], [13]-[22], [32]-[34], the well-established hybrid method consists of: (i) a bottom-up approach that is based on the MTL theory and eigenvalue decomposition (EVD) decomposition for the single-input single-output (SISO) systems of this paper; and (ii) a top-down approach that is denoted as TM2 method and is based on the concatenation of multidimensional chain scattering matrices. Through the original version of TM2 method, the hybrid method gives as outputs the corresponding modal transfer functions and modal reflection coefficients when the OV MV MTL configuration and OV MV BPL topology are given as inputs to the hybrid method.

On the basis of the applied coupling scheme, which is the practical way that the signals are injected into OV MV lines and the outputs of the hybrid method, coupling transfer functions and coupling reflection coefficients can be determined. In fact, two main categories of coupling schemes are mainly supported by the OV MV BPL networks, namely [4], [15], [17], [23], [24], [35]-[37]: (i) Wire-to-Ground (WtG) coupling schemes; and (ii) Wire-to-Wire (WtW) coupling schemes. In the case of $\mathrm{WtG}$ coupling schemes, which are examined in this paper, the $\mathrm{WtG}$ coupling transfer function $H^{\mathrm{WtG}}\{\}$ is given from

$$
H^{\mathrm{WtG}^{\mathrm{s}}}\{\cdot\}=\left[\mathbf{C}^{\mathrm{WtG}}\right]^{\mathrm{T}} \cdot \mathbf{T}_{\mathrm{V}} \cdot \mathbf{H}^{\mathrm{m}}\{\} \cdot \mathbf{T}_{\mathrm{V}}^{-1} \cdot \mathbf{C}^{\mathrm{WtG}}
$$

while $\mathrm{WtG}$ coupling reflection coefficient $\Gamma^{\mathrm{Wto}}\{\cdot\}$ is determined by

$$
\Gamma^{\mathrm{WtG}}\{\}=\left[\mathbf{C}^{\mathrm{WtG}}\right]^{\mathrm{T}} \cdot \mathbf{T}_{\mathrm{V}} \cdot \boldsymbol{\Gamma}_{\mathrm{in}}^{\mathrm{m}}\left\{\{\} \cdot \mathbf{T}_{\mathrm{V}}^{-1} \cdot \mathbf{C}^{\mathrm{WtG}}\right.
$$

where $\mathbf{C}^{\mathrm{WtG}}$ is an $n^{\mathrm{OVMv}} \times 1$ coupling column vector with zero elements except in row $s$ where the value is equal to $1, \mathrm{~T}_{\mathrm{V}}$ is a $n^{\mathrm{OVMV}} \times n^{\text {ovmv }}$ matrix that depends on the frequency, the OV MV MTL configuration and the physical properties of the cables, $\mathbf{H}^{\mathrm{m}}\{\}$ is the $n^{\text {ovMv }} \times n^{\text {ovmv }}$ EVD modal transfer function matrix and $\Gamma_{\text {in }}^{\mathrm{m}}\{\}$ is the $n^{\text {ovmv }} \times n^{\text {ovMv }}$ EVD modal reflection coefficient matrix. Both EVD modal transfer function matrix and EVD modal reflection coefficient matrix are given as outputs by the TM2 method [6]-[10], [13]-[17], [20], [28], [38].

To receive the WtG coupling transfer function and reflection coefficient of eqs. (1) and (2), respectively, certain assumptions for the circuital parameters of OV MV BPL topologies need to be taken into account during their determination of the original TM2 method. In accordance with [4], these assumptions are: (i) The branch lines are assumed identical to the main distribution ones; (ii) The interconnections between the main distribution and branch conductors of the lines are all connected; (iii) The transmitting and the receiving ends are assumed matched to the characteristic impedance of the modal channels; and (iv) The branch terminations are assumed open circuits.

\subsection{Faults and Instabilities in OV MV BPL Topologies}


During the continuous operation of the distribution power grid, critical problematic conditions can occur across it whose nature differs from the measurement differences. The presence of these problematic conditions endangers power quality and power distribution safety while these conditions are divided into two categories, namely: faults and instabilities [23]-[27]. Fault category, which describes all the interruptions that can occur in the lines of a distribution power grid, comprises two subcategories, say: main distribution line faults and branch line faults. Main distribution line faults that are of the interest of these three papers describe the condition where a main distribution line is interrupted due to physical or human reasons [27]. Main distribution line faults can be assumed to behave as either short- or open-circuit terminal loads. With reference to Fig. 1(b), let the main distribution line be broken at the position $\sum_{i=1}^{k} L_{k}+L_{\mathrm{m}, \mathrm{f}}$ from the transmitting end. A critical incident that determines the presence of this fault is the immediate communications failure between the transmitting and receiving end. However, there is a number of reasons why an immediate communications failure may appear in an OV MV BPL network thus creating a cause ambiguity. In addition, even if a main distribution line fault occurs and is identified, the localization of the exact fault position can significantly facilitate the maintenance personnel. Various efforts concerning the main distribution line fault localization have already been presented in [31], [39]-[42]. In this paper, the identification of a main distribution line fault is secured via the study of the behavior of reflection coefficients that come from the hybrid method.

From the third assumption of Sec.IIC, which concerns the circuital parameters of the original TM2 method, it is assumed that the terminal load is matched to the characteristic impedance of the modal channels but this is a not valid assumption when a main distribution line fault arises. Since main distribution line faults can be assumed to behave as either short- or open-circuit terminal loads, the original TM2 method fails to handle this situation in terms of the occurred reflection coefficients. The extension of TM2 method to cope with the aforementioned load terminations in terms of the reflection coefficients is described in Sec.III.

\section{Original and Extended TM2 Method}

Based on the model description of [17], the original TM2 method is extended in this paper by exploiting the generic multidimensional network analysis of [21], [34]. This extension copes with the different terminal loads that may occur during a main distribution line fault. The extended TM2 method is suitable for both transmission and distribution BPL networks so that the respective main transmission and distribution line faults can be handled and, thus, simulated.

In accordance with the definition of the original TM2 method of [17] and with reference to Fig. 1(a), an end-to-end BPL topology is separated into network modules, each of them comprising the successive branches encountered. BPL signal transmission through the serial connection of the various network modules is taken into account through the concatenation of their respective chain scattering matrices. Each network module may be considered as a cascade of two submodules, say: (i) the "transmission" submodule representing a distribution line of length $L_{k}$; and (ii) a "shunt" submodule representing the cascade of the branch termination $A_{k}^{\prime}$, the 
branch line of length $L_{\mathrm{b} k}$ and the interconnection between the main and branch conductors. Based on the specialized algebra for handling multidimensional scattering matrices, which is analytically presented in [17], [21], [34], the $2 n^{\text {OVMV }} \times 2 n^{\text {OVMV }}$ chain scattering matrix of the network module $\mathbf{T}^{k}$ is determined by using the appropriate cascade rule order. The last module of the BPL topology is the distribution line of length $L_{N+1}$ characterized by its $2 n^{\text {OVMV }} \times 2 n^{\text {OVMV }}$ chain scattering matrix $\mathbf{T}^{N+1}$. Having determined the chain scattering matrices of the various network modules encountered along the end-to-end connection, the $2 n^{\text {OVMV }} \times 2 n^{\text {OVMV }}$ overall end-to-end chain scattering matrix of the original TM2 method is evaluated through the multiplication rule from

$$
\mathbf{T}^{\text {overall, original }}=\left[\begin{array}{c:c}
\mathbf{T}_{11}^{\text {overall, original }} & \mathbf{T}_{12}^{\text {overall, original }} \\
\hdashline \mathbf{T}_{21}^{\text {overall, onginal }} & \mathbf{T}_{22}^{\text {overall, original }}
\end{array}\right]=\prod_{k=1}^{N+1} \mathbf{T}^{k}
$$

where $\quad \mathbf{T}_{11}^{\text {overall,original }}, \mathbf{T}_{12}^{\text {overall,original }}, \quad \mathbf{T}_{21}^{\text {overall,original }}$ and $\mathbf{T}_{22}^{\text {overall,original }}$ are the $n^{\text {OVMV }} \times n^{\text {OVMV }}$ matrix elements of the $\mathbf{T}^{\text {overall,orginal }}$ as evaluated from eq. (3). The respective $2 n^{\text {OVMV }} \times 2 n^{\text {OVMV }}$ overall end-to-end scattering matrix is obtained from [17], [21], [34]

$$
\begin{aligned}
& \mathbf{S}^{\text {overall, original }}=\left[\begin{array}{l:l}
\mathbf{S}_{11}^{\text {overall, original }} & \mathbf{S}_{12}^{\text {overall, original }} \\
\hdashline \mathbf{S}_{21}^{\text {overall,original }} & \mathbf{S}_{22}^{\text {overall,original }}
\end{array}\right]= \\
& =\left[\begin{array}{c:c}
\mathbf{T}_{21}^{\text {overall, original }}\left[\mathbf{T}_{11}^{\text {overall,onginal }}\right]^{-1} & \mathbf{T}_{22}^{\text {overall, original }}-\mathbf{T}_{21}^{\text {overall, original }}\left[\mathbf{T}_{12}^{\text {overall, original }}\right]^{-1} \mathbf{T}_{11}^{\text {overall,onginal }} \\
\hdashline\left[\mathbf{T}_{11}^{\text {overall,original }}\right]^{-1} & -\left[\mathbf{T}_{12}^{\text {overall,original }}\right]^{-1} \mathbf{T}_{11}^{\text {overall,orinal }}
\end{array}\right]
\end{aligned}
$$

where $\mathbf{S}_{11}^{\text {overall,original }}, \mathbf{S}_{12}^{\text {overall,original }}, \mathbf{S}_{21}^{\text {overall,original }}$ and $\mathbf{S}_{22}^{\text {overall,original }}$ are the $n^{\text {ovMV }} \times n^{\text {ovMV }}$ elements of the $\mathbf{S}^{\text {overall,original }}$ matrix as defined in eq. (4). Combining eqs. (1) and (4), the $n^{\text {OVMV }} \times n^{\text {OVMV }}$ EVD modal transfer function matrix is given by the $\mathbf{S}_{21}^{\text {overall,original }}$ element of the $\mathbf{S}^{\text {overall,original }}$ matrix, that is

$$
\mathbf{H}^{\mathrm{m} \text {,original }}\{\cdot\}=\mathbf{S}_{21}^{\text {overall, original }}=\left[\mathbf{T}_{11}^{\text {overall, original }}\right]^{-1}
$$

while the $n^{\text {OVMV }} \times n^{\text {OVMV }}$ EVD modal reflection coefficient matrix is given by

$$
\boldsymbol{\Gamma}_{\text {in }}^{\text {m, original }}\{\cdot\}=\mathbf{S}_{11}^{\text {overall, original }}
$$

Here, it should be noted that the transmitting and receiving ends are assumed matched to the characteristic impedance of the modal channels during the determination of the aforementioned modal quantities. Indeed, this is the suitable assumption for the normal operation of OV MV BPL networks. However, the terminal loads differentiate from the matched termination when a main distribution line fault occurs.

On the basis of the specialized algebra for handling the various branch terminations and terminal loads [21], [34], extended TM2 method transforms the overall end-to-end chain scattering matrix of the original TM2 method, which is given in eq. (4), into the $n^{\text {OVMV }} \times n^{\text {OVMV }}$ EVD modal reflection coefficient matrix $\boldsymbol{\Gamma}_{\text {in }}^{\text {mextended }}$ that is determined by

$\boldsymbol{\Gamma}_{\text {in }}^{\text {m,extended }}\{\cdot\}=\mathbf{S}_{11}^{\text {overall,original }}+\left[\mathbf{S}_{12}^{\text {overall,original }} \cdot\left(\mathbf{I}_{n^{\text {ovMV }}}-\boldsymbol{\Gamma}_{\text {out }}^{\mathrm{m} \text {,extended }} \cdot \mathbf{S}_{22}^{\text {overall, original }}\right)^{-1} \cdot \boldsymbol{\Gamma}_{\text {out }}^{\mathrm{m} \text {,extended }} \cdot \mathbf{S}_{21}^{\text {overall,original }}\right]$ 
where $\mathbf{I}_{n \text { ovmv }}$ is a $n^{\text {OvMv }} \times n^{\text {OvMv }}$ identity matrix and $\Gamma_{\text {out }}^{\text {mextended }}$ is the $n^{\text {ovMV }} \times n^{\text {OVMV }}$ EVD modal reflection coefficient matrix of the terminal load. From the observation of eq. (7), $\boldsymbol{\Gamma}_{\text {in }}^{\text {mextended }}$ degenerates into $\mathbf{S}_{11}^{\text {overall,original }}$ during the normal operation of the OV MV BPL network since then the terminal load can be assumed matched to the characteristic impedance of the modal channels, which implies that $\Gamma_{\text {out }}^{\text {m,extended }}$ is equal to a $n^{\text {ovmv }} \times n^{\text {ovMv }}$ zero matrix.

On the basis of eq. (7), the conversion of the original TM2 method to the extended TM2 method is also schematically given in Figs. 2(a) and (b). Comparing Fig. 2(a), which describes the output of the original TM2 method, and Fig. 2(b), which describes the output of the extended TM2 method, it is evident that the extended TM2 method assesses the reflection of the incident waves $\mathbf{a}_{1}$ and reflected waves $\mathbf{b}_{1}^{\prime}$ at the transmitting end through the modal reflection coefficient matrix $\boldsymbol{\Gamma}_{\mathrm{in}}^{\mathrm{m}, \mathrm{extended}}$ whereas it does not provide any transfer function details because no incident and reflected waves occur after the terminal load in contrast with the original TM2 method where incident waves $\mathbf{a}_{2}$ and reflected waves $\mathbf{b}_{2}$ appear at the receiving end and, afterwards, are measured.

As already been mentioned, the terminal load can be assumed matched to the characteristic impedance of the modal channels during the normal operation of the OV MV BPL networks whereas the terminal load may behave as either short- or open-circuit during a main distribution line fault depending on the location of the conductors of the main distribution lines after the fault. In order to assess and compare the previous behaviors in terms of their reflection coefficients when original and extended TM2 method is applied, $\boldsymbol{\Gamma}_{\mathrm{in}}^{\mathrm{m} \text {,original }}$ is given by eq. (6) during the normal operation whereas $\Gamma_{\mathrm{in}}^{\mathrm{m}, \text { extended }}$ is given by eq. (7) during the main distribution line fault condition. In the latter case, $\Gamma_{\text {out }}^{\mathrm{m} \text {,extended }}$ is assumed to be equal to $-\mathbf{I}_{n}$ ovmv (short-circuit terminal load) or $\mathbf{I}_{n^{\text {ovmv }}}$ (open-circuit terminal load) in order to evaluate $\boldsymbol{\Gamma}_{\mathrm{in}}^{\mathrm{m} \text {,extended }}$.

Summarizing the findings of this Section, the "real-life" operation of the OV MV BPL networks depends on the deployed coupling scheme systems across them. Since all the required modal quantities have been defined for the normal and fault operation by appropriately applying original and extended TM2 method, WtG coupling transfer function and $\mathrm{WtG}$ coupling reflection coefficient can easily be determined by eqs. (1) and (2), respectively, by appropriately replacing these modal quantities as previously outlined. 

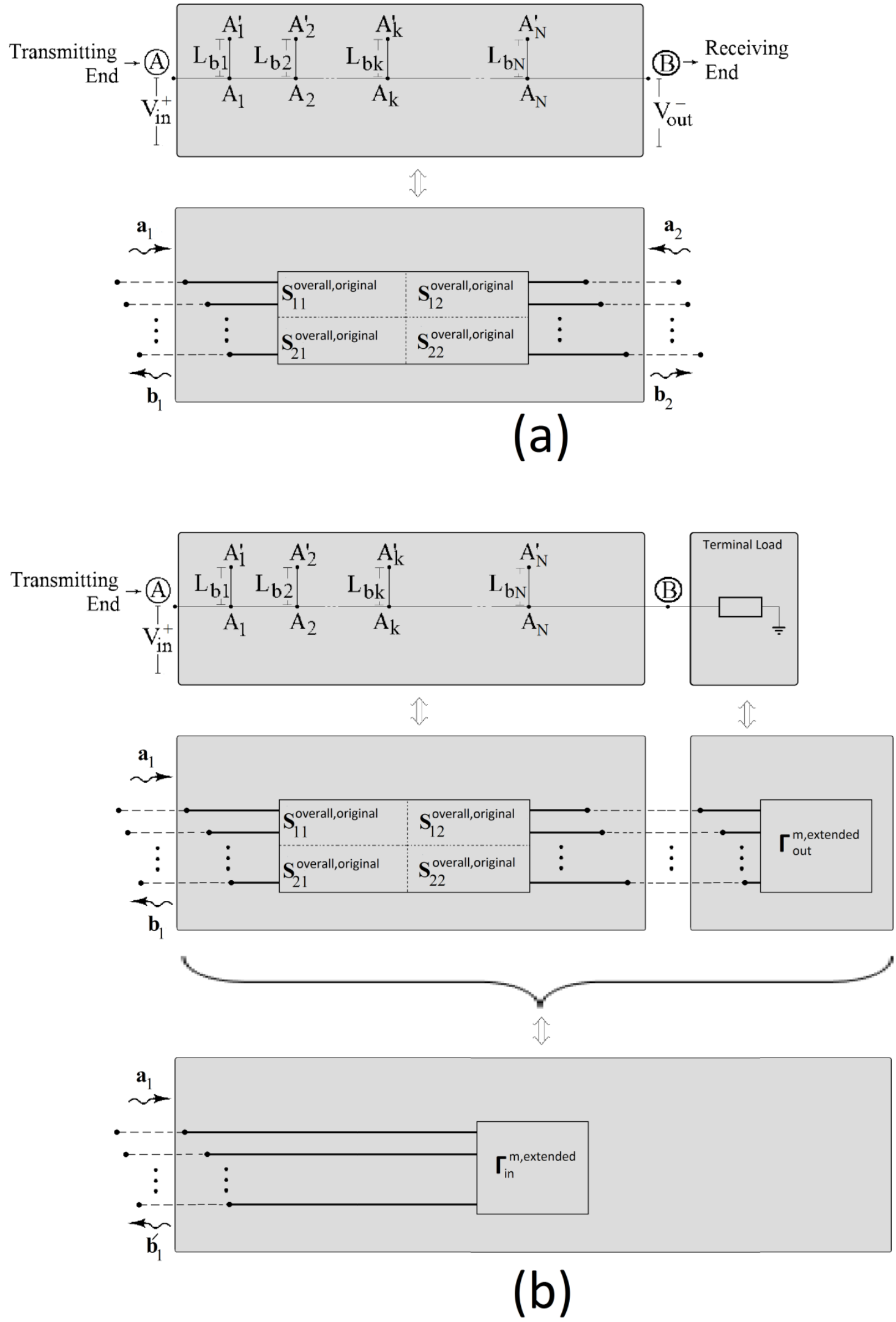

Figure 2. (a) Overall end-to-end scattering matrix of the original TM2 method. (b) Modal reflection coefficient matrix of the extended TM2 method when terminal load occurs at the receiving end. 


\section{Numerical Results and Discussion}

\subsection{Simulation Goals and Parameters}

The indicative topologies of OV MV BPL networks are simulated with the purpose of identifying a main distribution line fault by comparing the results of reflection coefficient of the original TM2 method with the ones of the extended TM2 method. The behavior of reflection coefficients is further detailed for the different terminal loads (i.e., short- or open-circuit termination) when a main distribution line fault occurs.

As regards the simulation specifications, those are the same with [4], [23]-[27]. More specifically, the BPL frequency range and flat-fading subchannel frequency spacing are assumed equal to $1-30 \mathrm{MHz}$ and $1 \mathrm{MHz}$, respectively. Therefore, the number of subchannels is equal to 30 in the examined frequency range. Arbitrarily, the $\mathrm{WtG}^{3}$ coupling scheme is applied during the following simulations. As it is usually done [10], [13], [14], [16], [18], [23], [24], [43], the selection of representative coupling schemes is a typical procedure for the sake of reducing manuscript size.

\subsection{Coupling Transfer Function and Coupling Reflection Coefficient for the Indicative OV MV BPL Topologies (Original TM2 Method)}

Prior to study the behavior of OV MV BPL networks when a main distribution line fault occurs, the magnitude of coupling transfer function and coupling reflection coefficient of the indicative OV MV BPL topologies is outlined when their terminal loads are assumed matched to the modal characteristics impedances. The nature of the studied terminal loads implies that original TM2 method is applied during the following simulations. Note that the behavior of the coupling reflection coefficient during the normal operation, which is presented in this subsection, is going to act as the benchmark in order to identify the existence of a main distribution line fault (see Sec.IVD).

In Fig. 3, the coupling transfer function is plotted versus frequency for the four indicative $\mathrm{OV} \mathrm{MV}$ BPL topologies of Sec.IIB when $\mathrm{WtG}^{3}$ coupling scheme is applied. In Fig. 4, similar curves with Fig. 3 are shown but for the magnitude of coupling reflection coefficient.

From Fig. 3, it is clear that the existence of branches encountered across the BPL signal transmission in the examined OV MV BPL topologies imposes spectral notches in the coupling transfer functions, which are superimposed to the relatively steady "LOS" transfer function. The depth and the extent of these spectral notches mainly depend on the number and the electrical length of the branches as well as the nature of branch terminations. As concerns the characteristics of branches, OV MV BPL topologies with high number of branches and relatively low branch electrical length, such as the examined urban case one, create hostile and aggravated multipath environments for the BPL signal transmission. Conversely, when the presence of branches is scarce and the branch length is high, transfer function of these OV MV BPL topologies, such as the examined OV MV BPL rural one, tends to converge to the behavior of the "LOS" case where shallow and rare spectral notches are observed. In all the other OV MV BPL topology cases, the behavior of their transfer functions lies between the one of urban (worst case) and "LOS" (best case) [6], [7], [8], [9]. Anyway, the challenge of mitigating these horrible transmission characteristics push the recent 


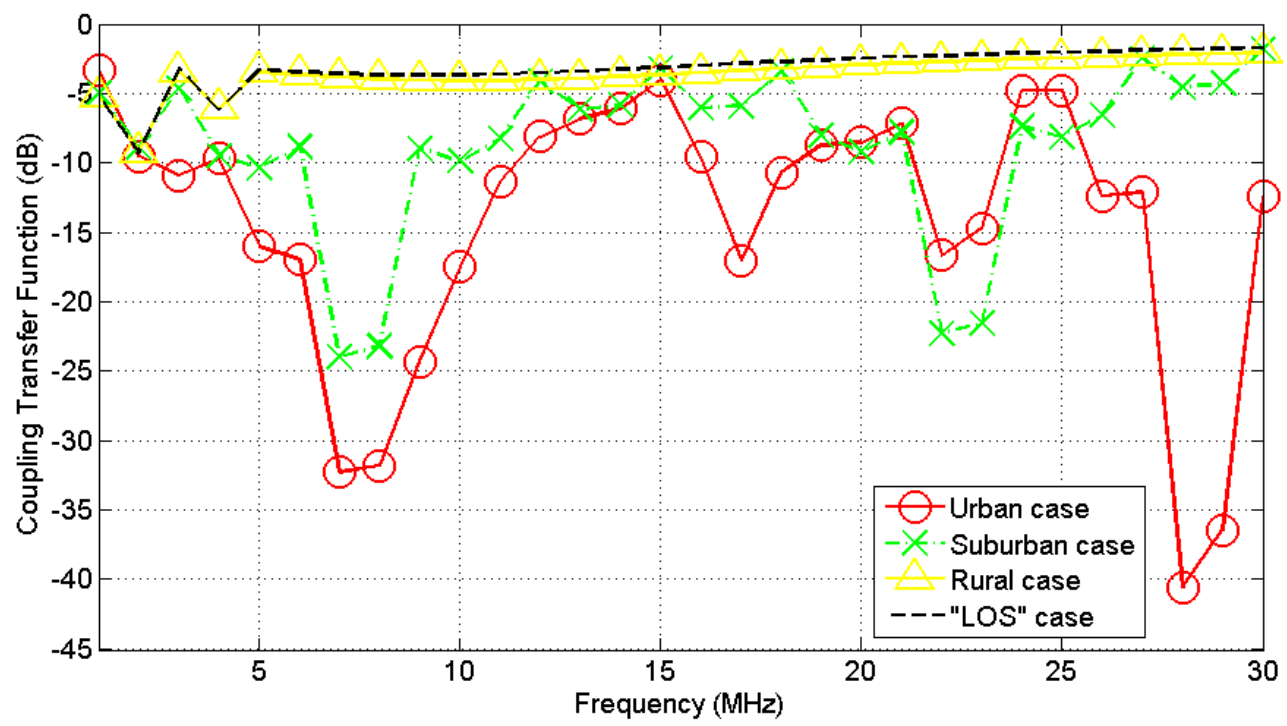

Figure 3. Coupling transfer function versus the frequency for the indicative OV MV BPL topologies when $\mathrm{WtG}^{3}$ coupling scheme is applied and normal operation conditions are assumed (the frequency spacing is equal to $1 \mathrm{MHz}$ ).

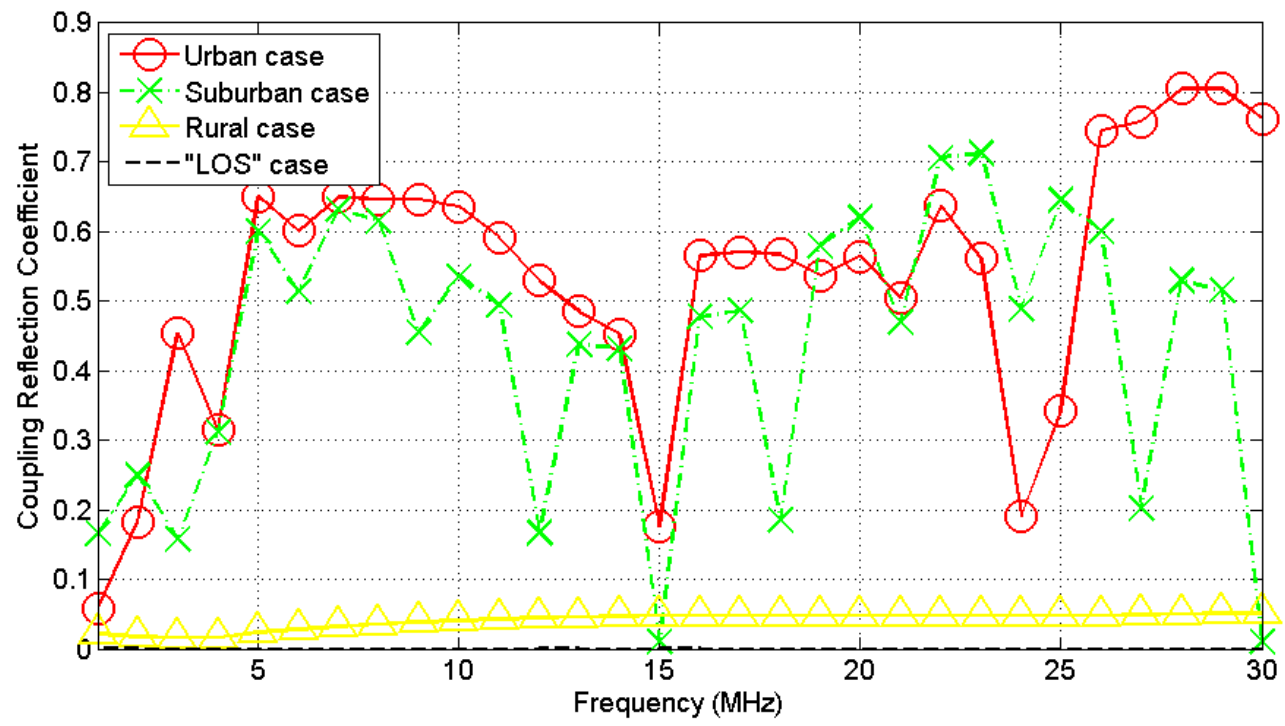

Figure 4. Coupling reflection coefficient versus the frequency for the indicative OV MV BPL topologies when $\mathrm{WtG}^{3}$ coupling scheme is applied and normal operation conditions are assumed (the frequency spacing is equal to $1 \mathrm{MHz}$ ).

research efforts towards communications solutions such as multi-hop repeater systems, multiple-input multiple-output consideration of the BPL channels and various resource allocation schemes [11], [18], [44], [45].

Similarly to coupling transfer functions, the magnitude of coupling reflection coefficients of the indicative OV MV BPL topologies present significant fluctuations in comparison with the almost zero reflection coefficient of the "LOS" case (matched termination load). In fact, the branch presence along the transmission path creates a 
spectral environment that resembles to that of power dividers [10], [32], [33]. Since different number and length of branches are connected to the main distribution line, this has as a result that the input impedance at the transmitting end presents a frequency-dependent behavior, which further affects the reflection coefficient at the same point. In general terms, the spectral behavior of the reflection coefficient can be approached in a similar way with the behavior of the transfer function; say, topologies with high number of branches having relatively short lengths of branches superimpose significant reflection and spectral notches to the "LOS" case whereas topologies with low number of branches and long lengths tend to render their reflection coefficient similar to the "LOS" case ones.

Observing both Figs. 3 and 4, it is obvious that the trend smoothness of transfer function and reflection coefficient curves, the extrema of the curves and the extent and depth of curve notches may act as an identity pattern for the OV MV BPL topologies. This unique property of the aforementioned curves is going to be exploited by the main line fault localization methodology (MLFLM) in the accompanying papers in order to localize the main distribution line faults that may occur in OV MV BPL networks [23]-[26].

\subsection{Coupling Reflection Coefficient for the Indicative OV MV BPL Topologies when Main Distribution Line Faults Occur (Extended TM2 Method)}

Already been mentioned, critical problematic conditions, such as the main distribution line faults, can occur across the distribution power grid during its operation. With reference to Fig. 1(b), let the main distribution line be broken at $750 \mathrm{~m}$ from the transmitting end. The four modified indicative OV MV BPL topologies are then differentiated as follows:

1. The modified urban topology (denoted as modified urban case) with $N=2$ branches $\left(L_{1}=500 \mathrm{~m}, L_{2}=200 \mathrm{~m}, L_{3}=50 \mathrm{~m}, L_{\mathrm{b} 1}=8 \mathrm{~m}, L_{\mathrm{b} 2}=13 \mathrm{~m}\right)$.

2. The modified suburban topology (denoted as modified suburban case) with $N=1$ branch $\left(L_{1}=500 \mathrm{~m}, L_{2}=250 \mathrm{~m}, L_{\mathrm{b} 1}=50 \mathrm{~m}\right)$.

3. The modified rural topology (denoted as modified rural case) with $N=1$ branch $\left(L_{1}=600 \mathrm{~m}, L_{2}=150 \mathrm{~m}, L_{\mathrm{b} 1}=300 \mathrm{~m}\right)$.

4. The "LOS" transmission along the same end-to-end distance $L=L_{1}+\ldots+L_{N+1}=750 \mathrm{~m}$ (denoted as modified "LOS" case) when no branches are encountered.

Here, it should be noted that the terminal load, which is located at the receiving end of the modified OV MV BPL topologies, is assumed equal to either short- or open-circuit. Hence, two sets of the four modified OV MV BPL topologies are examined in this subsection.

In accordance with Sec.III, the study of the behavior of OV MV BPL networks when a main distribution line fault occurs is focused on the examination of the reflection coefficients as dictated by eq. (7) through the application of the extended TM2 method. In Fig. 5, the magnitude of coupling reflection coefficient is plotted versus frequency for the four modified OV MV BPL topologies when $\mathrm{WtG}^{3}$ coupling scheme is applied and the terminal load is assumed to be short-circuit. In Fig. 6, similar curves with Fig. 5 are shown with the assumption that the terminal load is an open-circuit. 


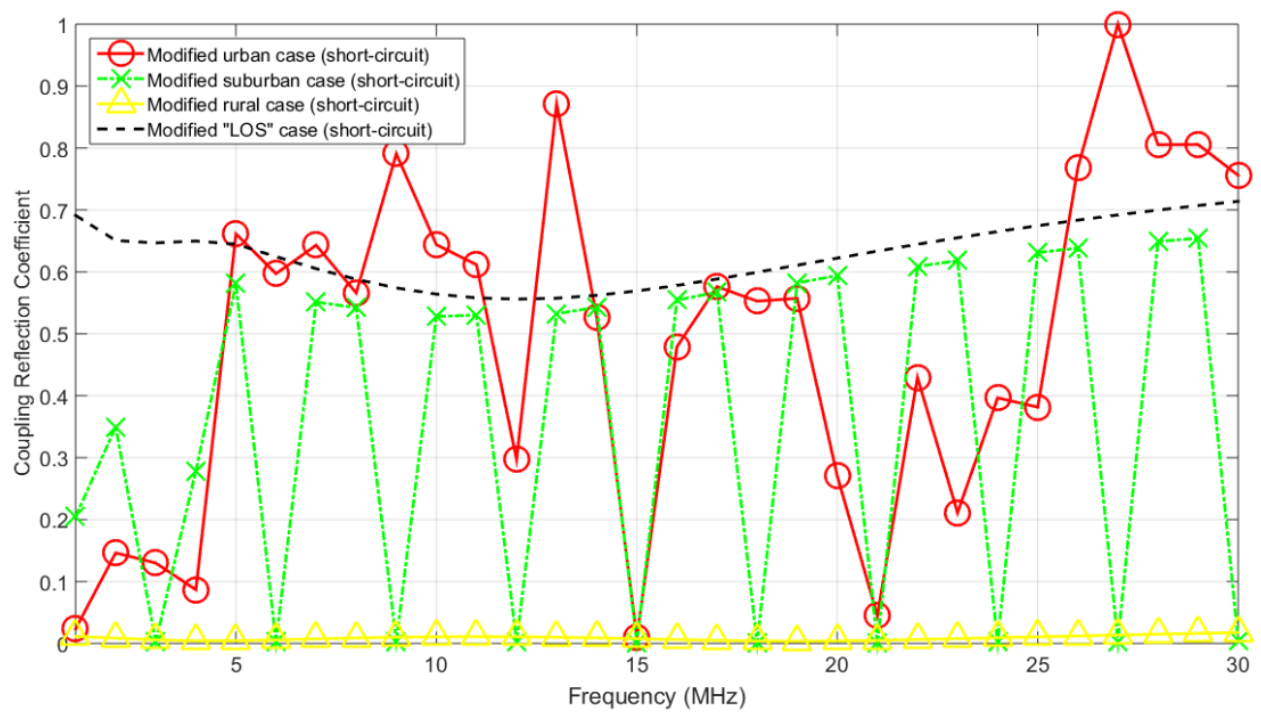

Figure 5. Coupling reflection coefficient versus the frequency for the modified OV MV BPL topologies when $\mathrm{WtG}^{3}$ coupling scheme is applied and short-circuit is assumed as the terminal load (the frequency spacing is equal to $1 \mathrm{MHz}$ ).

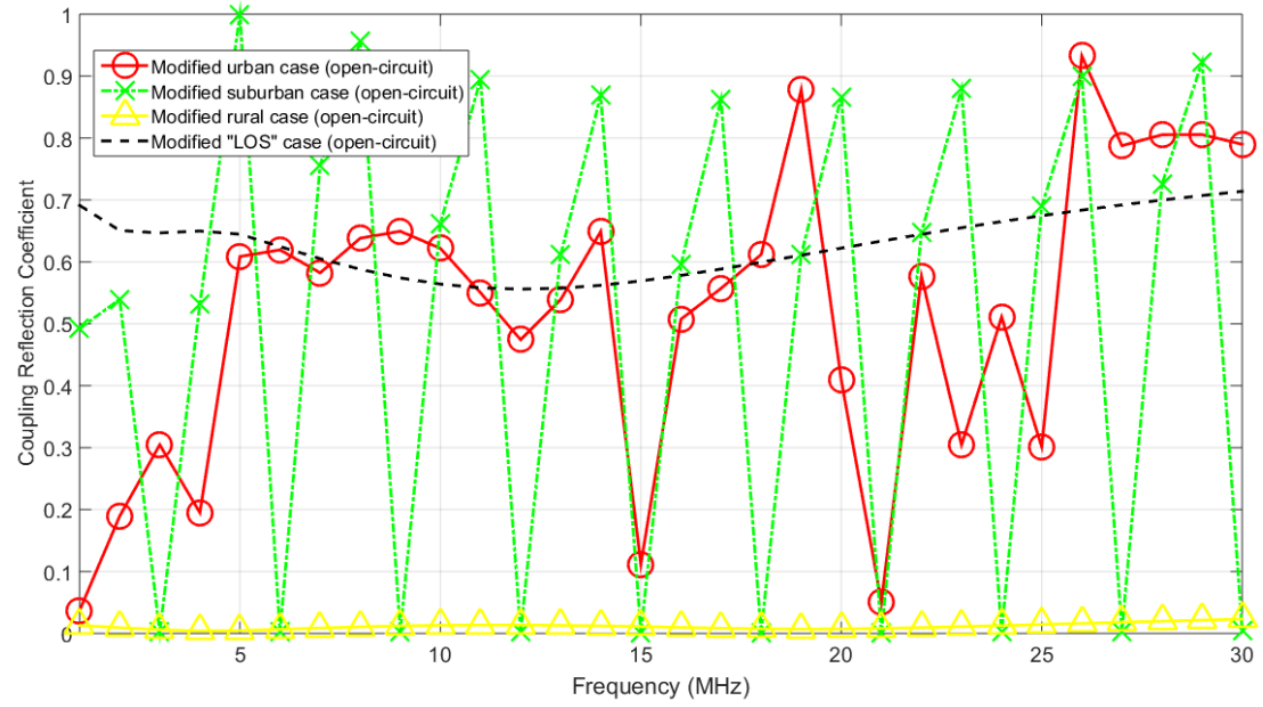

Figure 6. Same plots with Fig.5 but for an open-circuit terminal load.

The first fact that implies the presence of a main distribution line fault is the immediate communications failure between the transmitting and receiving end while the validation of the fault presence comes from the examination of the reflection coefficient at the transmitting end as highlighted in Figs. 4-6. In fact, the nature of the terminal load critically determines the form of the coupling reflection coefficient; say, any termination load, which differs from the matched terminal load of the normal operation of the OV MV BPL networks, significantly differentiates the coupling reflection coefficient from the one presented during the normal operation regardless of the examined topology. 
Comparing Figs. 4-6, it deserves special attention the behavior of the magnitude of the coupling reflection coefficient of the "LOS" case. First, the values of the reflection coefficient of "LOS" case drastically change from the zero when the terminal load connected at the receiving end takes a value that differs from the matched termination. Actually, the magnitudes of the reflection coefficient coincide when the terminal load is assumed to be either short- or open-circuit. Anyway, this is explained by eq. (7) and the values assigned to $\Gamma_{\text {out }}^{\mathrm{m} \text {,extended }}$ in Sec.III for each of the aforementioned terminal load cases.

From the aforementioned observations, it is evident that the identification and further localization of a main distribution line fault comes from the difference of reflection coefficients that occurs between the normal and fault operation, which is highlighted in the following subsection.

\subsection{Coupling Reflection Coefficient Differences between the Normal and Fault Operation of the Indicative OV MV BPL Topologies}

Already been reported in Sec.IVC, the main distribution line faults differentiate the reflection coefficient behavior between the normal and fault operation. In this subsection, a study is undergone focusing on the comparative behavior of OV MV BPL topologies during the main distribution line faults.

In Fig. 7, the reflection coefficient differences of the indicative OV MV BPL topologies between their normal and fault operation is plotted versus frequency when $\mathrm{WtG}^{3}$ coupling scheme is applied and the terminal load is assumed to be short-circuit termination. The main distribution line fault is located at $750 \mathrm{~m}$ from the transmitting end and the reflection coefficient difference, which is presented in Fig.7, essentially defines the difference between Figs. 5 and 6 for given OV MV BPL topology. In Figs. 8-10, same plots with Fig. 7 but for the main distribution line fault to be located at $1 \mathrm{~m}, 520 \mathrm{~m}$ and $910 \mathrm{~m}$, respectively. Similar curves with Figs. 7-10 are given in Figs. 11-14, but for the open-circuit terminal load case.

Examining Figs. 7-14, several interesting conclusions can be deduced:

- When the main distribution line fault is located immediately after the transmitting end, the impact of the presence of the transmission line is limited. Indeed, when the main distribution line fault is located at $1 \mathrm{~m}$ from the transmitting end, the reflection coefficient is equal to -1 or 1 if the terminal load is a short- or opencircuit termination, respectively. Therefore, the magnitude of the reflection coefficients is equal to 1 in both the cases. As presented in Figs. 8 and 12, it is expected that the coupling reflection coefficient difference of the "LOS" case is equal to -1 since the absolute value of the reflection coefficient of the "LOS" topology during its normal operation is equal to 0 .

- Apart from the "LOS" case where the main distribution line fault is located at the transmitting end, the coupling reflection coefficient differences of OV MV BPL topologies with branches present fluctuations that are distributed around the zero regardless of the terminal load.

- When a communications failure between the transmitting and receiving end persists and the coupling reflection coefficient differences insist on differing from zero in the frequency domain a main distribution line fault is present. 


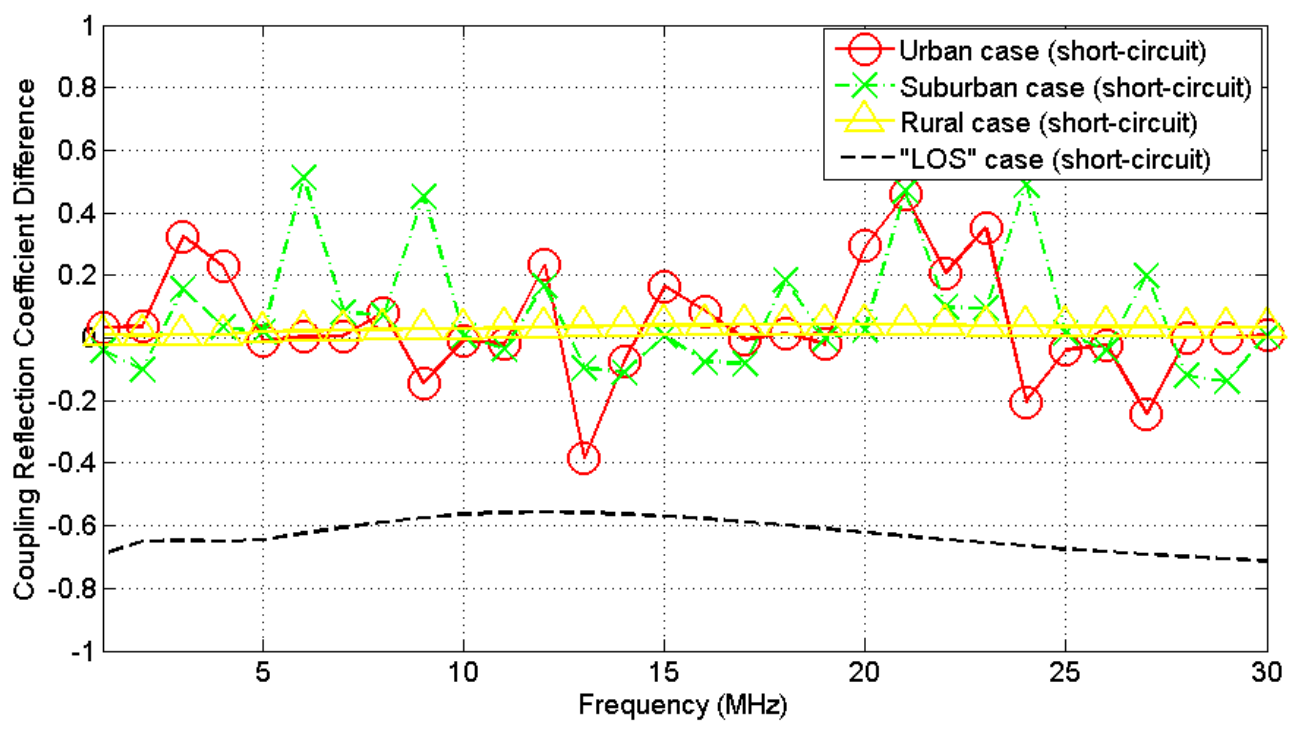

Figure 7. Coupling reflection coefficient difference versus the frequency between the original and modified indicative OV MV BPL topologies when $\mathrm{WtG}^{3}$ coupling scheme is applied and shortcircuit is assumed as the terminal load at $750 \mathrm{~m}$ from the transmitting end (the frequency spacing is equal to $1 \mathrm{MHz}$ ).

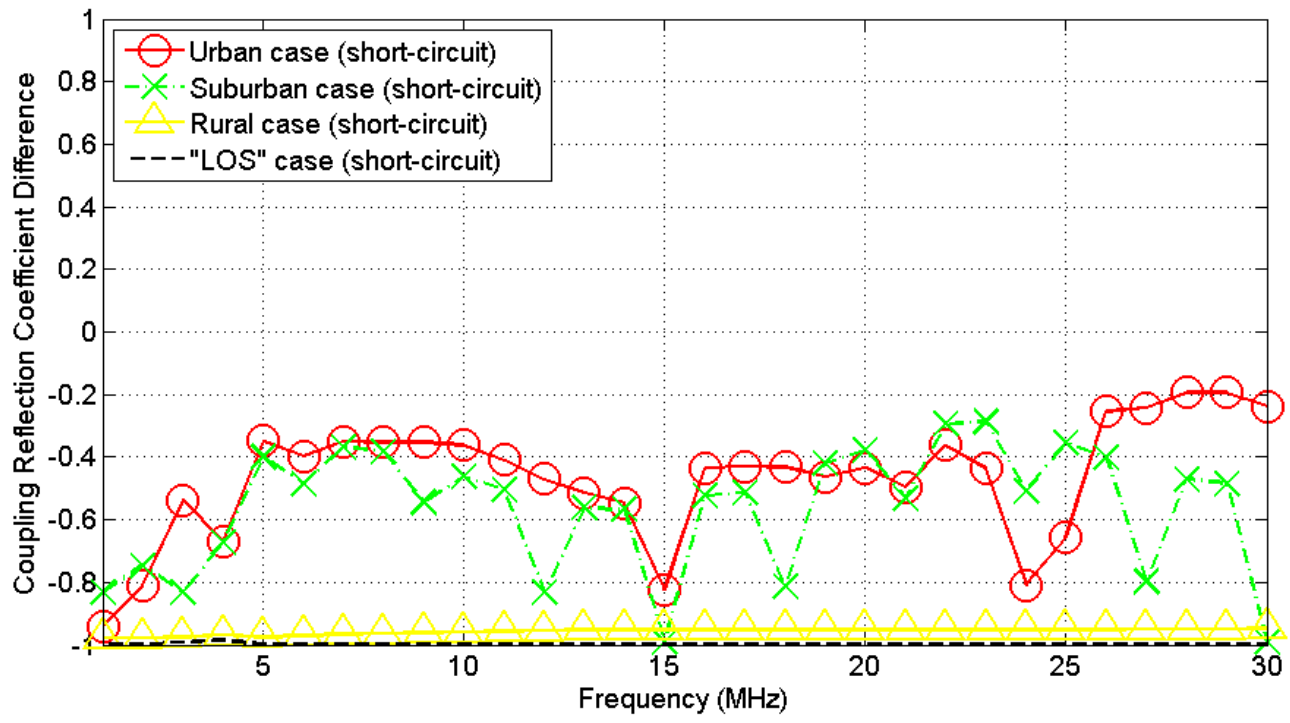

Figure 8. Coupling reflection coefficient difference versus the frequency between the original and modified indicative OV MV BPL topologies when $\mathrm{WtG}^{3}$ coupling scheme is applied and shortcircuit is assumed as the terminal load at $1 \mathrm{~m}$ from the transmitting end (the frequency spacing is equal to $1 \mathrm{MHz}$ ). 


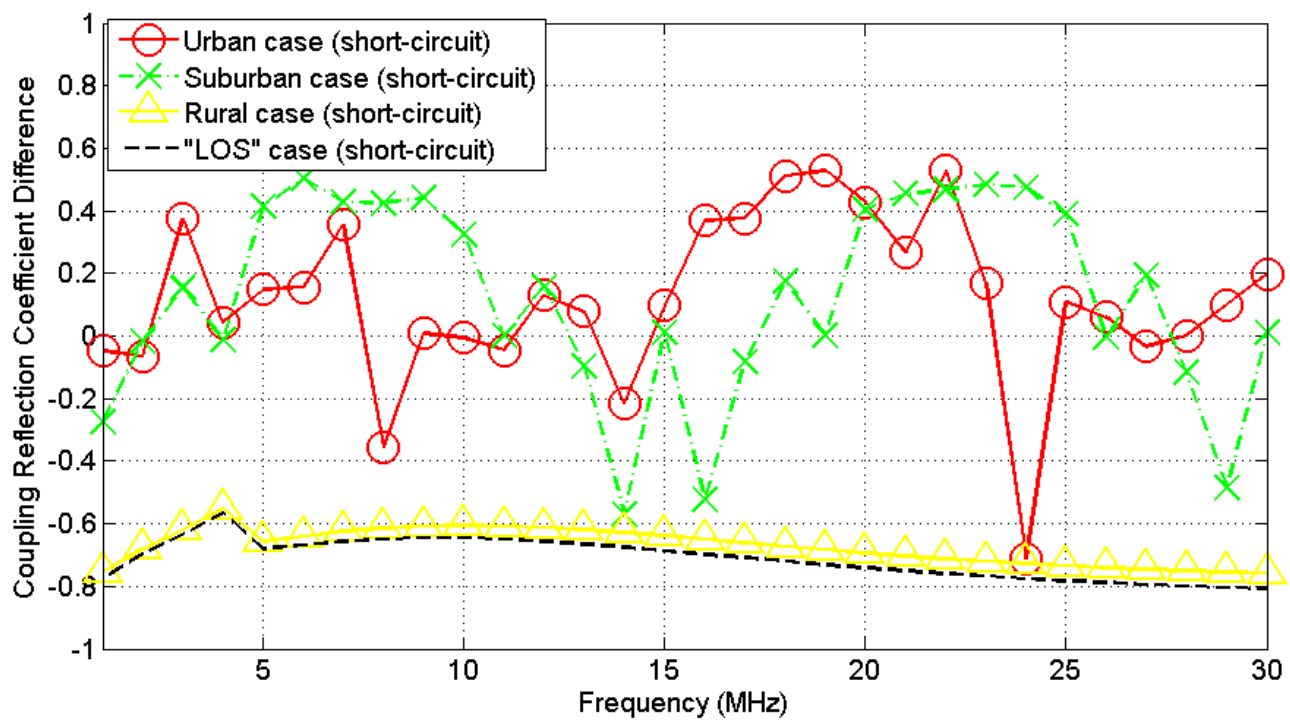

Figure 9. Coupling reflection coefficient difference versus the frequency between the original and modified indicative OV MV BPL topologies when $\mathrm{WtG}^{3}$ coupling scheme is applied and shortcircuit is assumed as the terminal load at $520 \mathrm{~m}$ from the transmitting end (the frequency spacing is equal to $1 \mathrm{MHz}$ ).

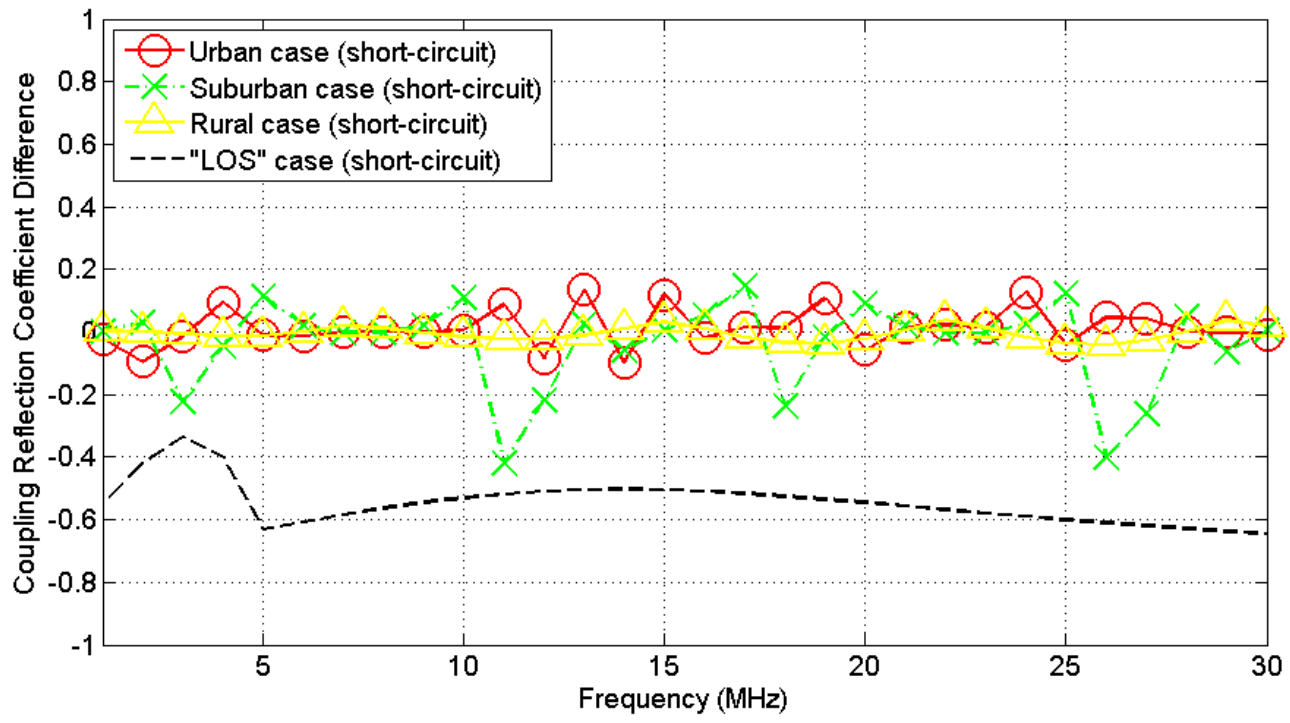

Figure 10. Coupling reflection coefficient difference versus the frequency between the original and modified indicative OV MV BPL topologies when $\mathrm{WtG}^{3}$ coupling scheme is applied and shortcircuit is assumed as the terminal load at $910 \mathrm{~m}$ from the transmitting end (the frequency spacing is equal to $1 \mathrm{MHz}$ ). 


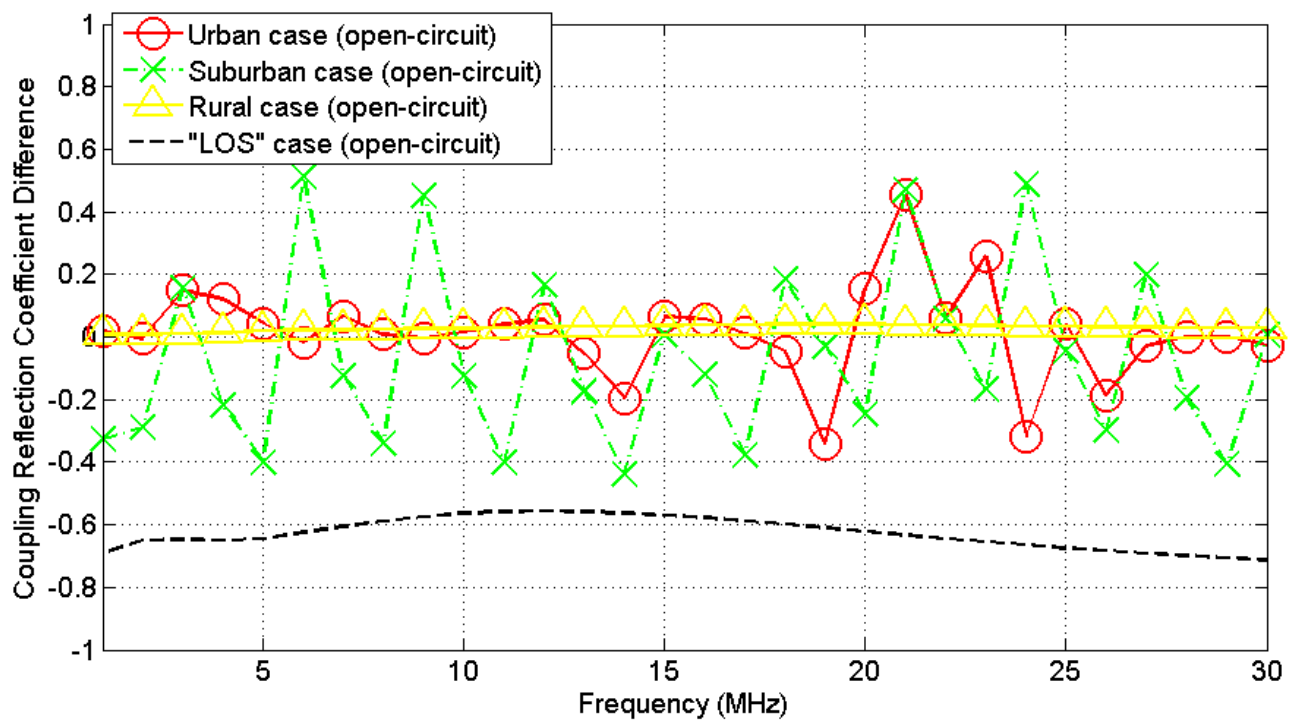

Figure 11. Same plots with Fig.7 but for an open-circuit terminal load.

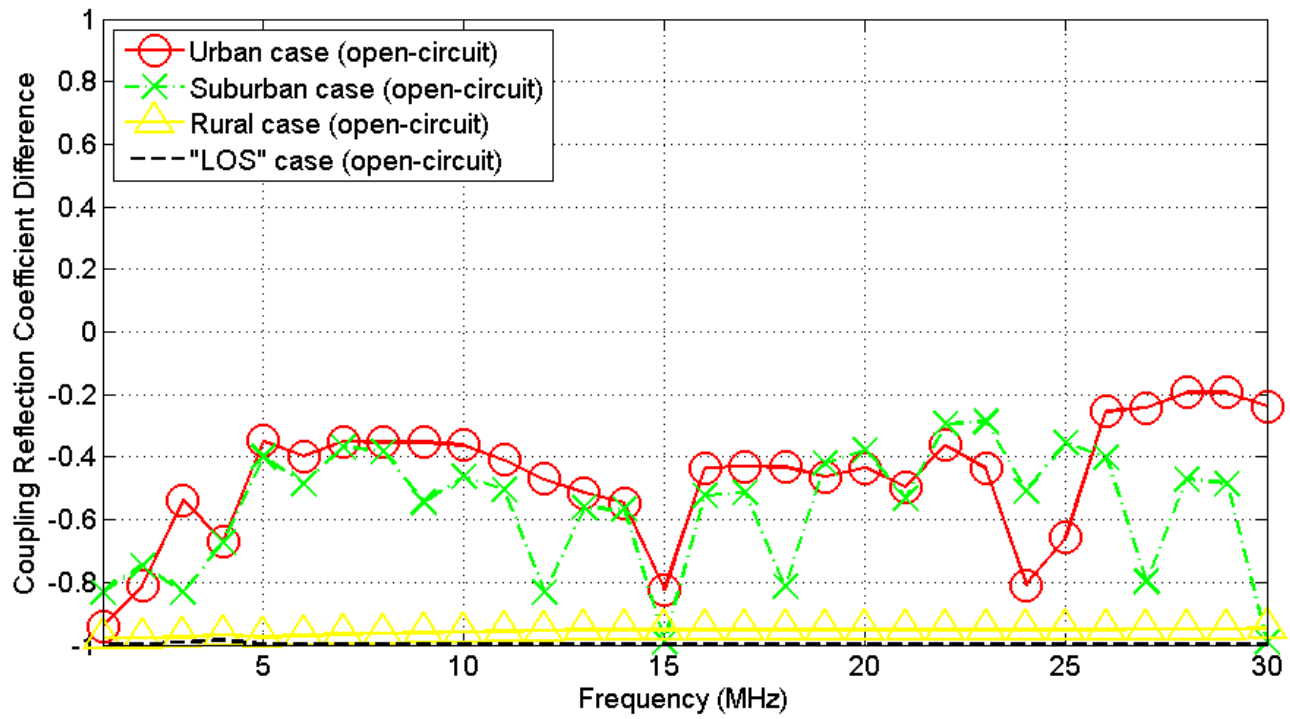

Figure 12. Same plots with Fig.8 but for an open-circuit terminal load. 


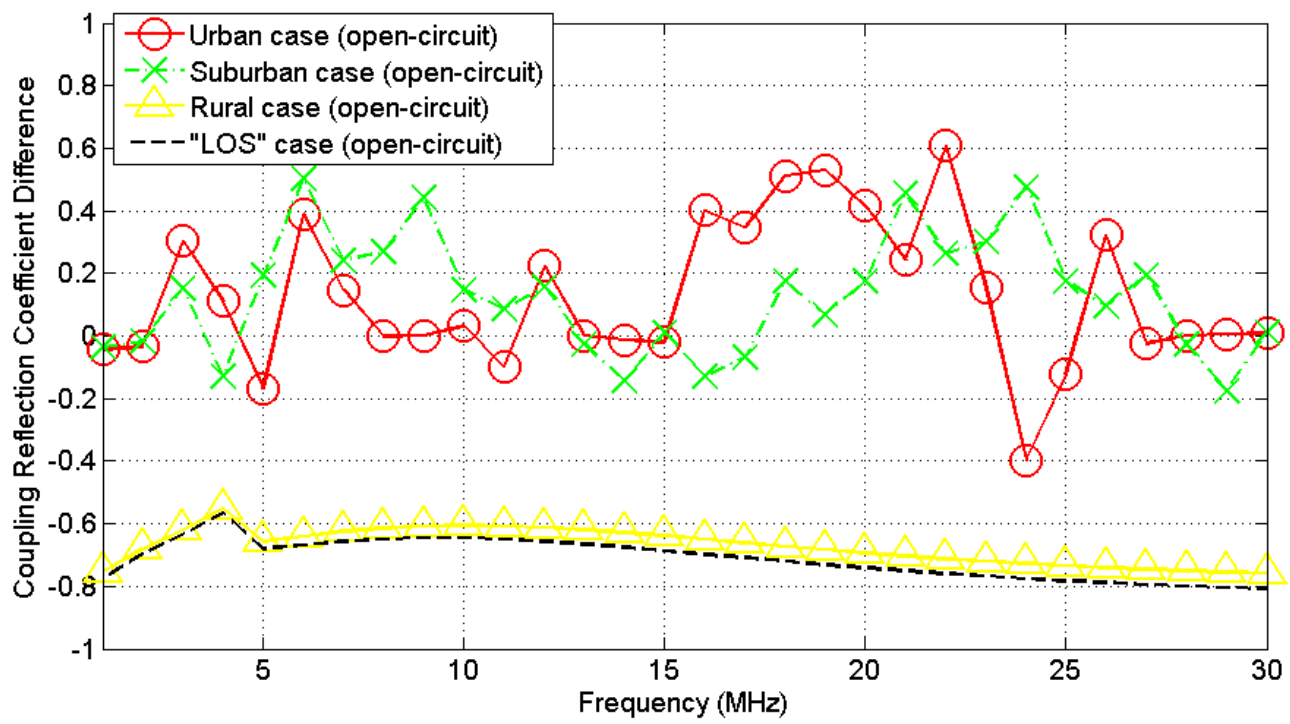

Figure 13. Same plots with Fig.9 but for an open-circuit terminal load.

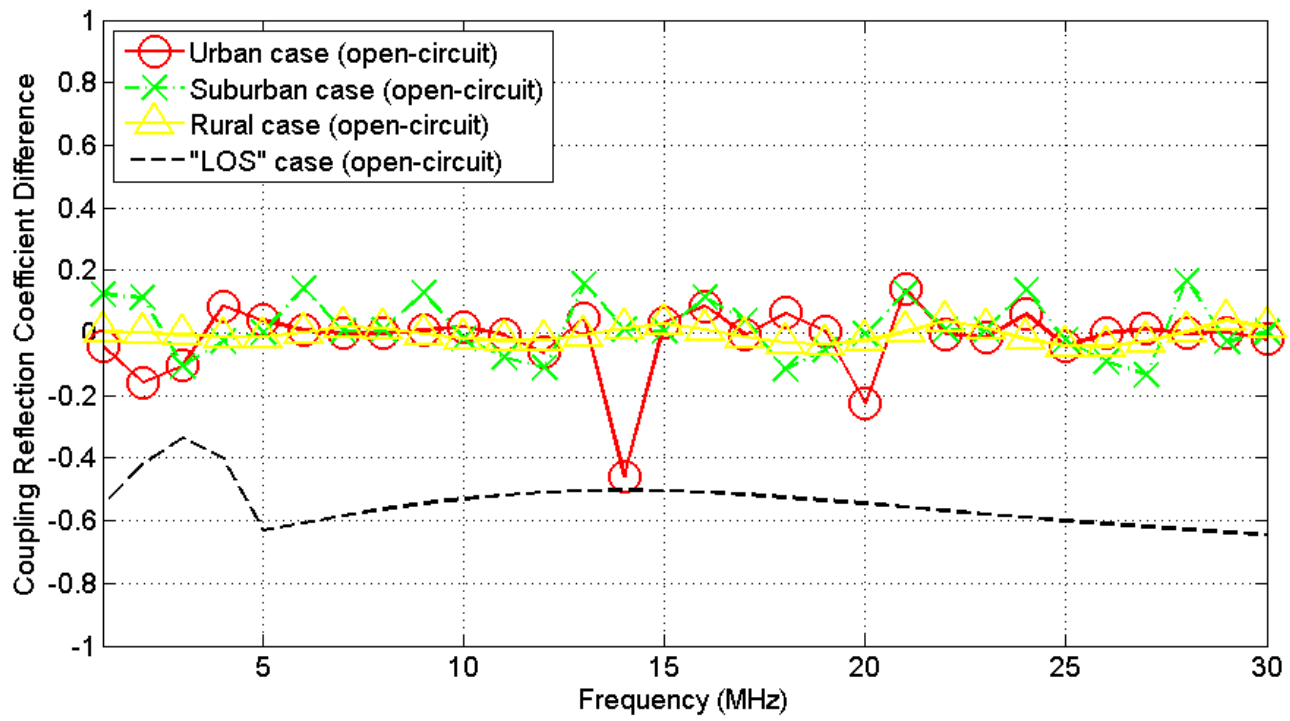

Figure 14. Same plots with Fig.10 but for an open-circuit terminal load.

Here, the identification of a main distribution line fault is secured and the localization of main distribution line faults through MLFLM procedure can be initiated (for more details concerning the localization of main distribution line faults and MLFLM, see [46]).

- The detection of a main distribution line fault is easier in the OV MV BPL topologies with more branches than in the "LOS" case since the fluctuations of the coupling reflection coefficient differences around zero get severe in the aggravated topology cases. In contrast, rural and "LOS" OV MV BPL topologies demand more attention when the identification of main distribution line faults is undergone since their fluctuations are milder. 
- Similarly to the findings regarding the unique appearance of transfer functions and reflection coefficients of OV MV BPL topologies, the unique characteristics of the coupling reflection coefficient differences (i.e., the trend of the curve, the extrema of the curves and the extent and depth of curve notches) are exploited as the identity pattern in order to exact localize main distribution line faults in [46], [47].

- Although the identification of a main distribution line fault has been theoretically secured in all the OV MV BPL topologies through the coupling reflection coefficient differences, the identification of these faults can be problematic in the "real-life" conditions since measurement differences can be added during the determination of reflection coefficients (fault alarm case). The countermeasures against the measurement differences by using piecewise monotonic data approximations (PMAs) as well as the identification efficiency of a main distribution line fault through the reflection coefficient differences is examined in [47].

- Apart from the identification of a main distribution line fault, the goal of these papers is the exact localization of the fault. On the basis of the PMA benchmark results of [47] and the extended TM2 method, TIM of [23] and FIIM of [24] can further be upgraded in order to deal with the main distribution line faults that constitute the only fault case which cannot be identified by TIM and FIIM in [25], [26]. MLFLM is going to exploit the unique characteristics of reflection coefficients of OV MV BPL topologies as well as the findings of this paper and [47] in order to exactly localize the main distribution line faults that may occur in OV MV BPL networks in [46].

\section{Conclusions}

In this first paper, the identification methodology of main distribution line faults in OV MV BPL networks has been presented and assessed. Initially, the extension of TM2 method has been analyzed on the basis of the original TM2 method through its generic multidimensional network analysis. In fact, the identification of main distribution line faults is based on the coupling reflection coefficients derived from the original and the extended TM2 method as well as their differences. The assessment of the identification of main distribution line faults has been applied to four indicative OV MV BPL topologies when their terminal loads are assumed to be either matched or shortcircuit or open-circuit. Actually, the pattern of the coupling reflection coefficients significantly depends on the examined OV MV BPL topology as well as the nature of the terminal loads, thus implying that there are significant differences in coupling reflection coefficients between the normal and fault condition for given OV MV BPL topology. Synoptically, the coupling reflection coefficient may act as an identity pattern for given OV MV BPL topology and terminal load while the communications failure between the transmitting and receiving end and the fluctuations of coupling reflection differences at the transmitting end marks the existence of a main distribution line fault across the $\mathrm{OV}$ MV BPL topology.

The influence of the measurements differences during the identification of main distribution line faults, the countermeasures against measurement differences by applying PMAs and the proposal of MLFLM with the intention to localize the main distribution line faults are going to be investigated in the following two papers. 


\section{CONFLICTS OF INTEREST}

The author declares that there is no conflict of interests regarding the publication of this paper.

\section{References}

[1] G. Kaddoum and N. Tadayon, "Differential Chaos Shift Keying: A Robust Modulation Scheme for Power-Line Communications," IEEE Trans. on Circuits and Systems II: Express Briefs, vol. 64, no. 1, pp. 31-35, 2017.

[2] A. Milioudis, G. Andreou, and D. Labridis, "Optimum transmitted power spectral distribution for broadband power line communication systems considering electromagnetic emissions," Elsevier Electric Power Systems Research, vol. 140, pp. 958-964, Nov. 2016.

[3] K. Sharma and L. M. Saini, "Power-line Communications for Smart Grid: Progress, Challenges, Opportunities and Status," Elsevier Renewable and Sustainable Energy Reviews, vol. 67, pp. 704-751, 2017.

[4] A. G. Lazaropoulos, "Best L1 Piecewise Monotonic Data Approximation in Overhead and Underground Medium-Voltage and Low-Voltage Broadband over Power Lines Networks: Theoretical and Practical Transfer Function Determination," Hindawi Journal of Computational Engineering, vol. 2016, Article ID 6762390, 24 pages, 2016. doi:10.1155/2016/6762390. [Online]. Available: https://www.hindawi.com/journals/jcengi/2016/6762390/cta/

[5] C. Cano, A.Pittolo, D. Malone, L. Lampe, A. M.Tonello, and A. Dabak, "State-ofthe-art in Power Line Communications: From the Applications to the Medium," IEEE J. Sel. Areas Commun.,vol. 34, pp. 1935-1952, 2016.

[6] A. G. Lazaropoulos and P. G. Cottis, "Transmission characteristics of overhead medium voltage power line communication channels," IEEE Trans. Power Del., vol. 24, no. 3, pp. 1164-1173, Jul. 2009.

[7] A. G. Lazaropoulos and P. G. Cottis, "Capacity of overhead medium voltage power line communication channels," IEEE Trans. Power Del., vol. 25, no. 2, pp. 723-733, Apr. 2010.

[8] A. G. Lazaropoulos and P. G. Cottis, "Broadband transmission via underground medium-voltage power lines-Part I: transmission characteristics," IEEE Trans. Power Del., vol. 25, no. 4, pp. 2414-2424, Oct. 2010.

[9] A. G. Lazaropoulos and P. G. Cottis, "Broadband transmission via underground medium-voltage power lines-Part II: capacity," IEEE Trans. Power Del., vol. 25, no. 4, pp. 2425-2434, Oct. 2010.

[10] A. G. Lazaropoulos, "Broadband transmission characteristics of overhead highvoltage power line communication channels," Progress in Electromagnetics Research B, vol. 36, pp. 373-398, 2012. [Online]. Available: http://www.jpier.org/PIERB/pierb36/19.11091408.pdf

[11] A. G. Lazaropoulos, "Capacity Performance of Overhead Transmission MultipleInput Multiple-Output Broadband over Power Lines Networks: The Insidious Effect of Noise and the Role of Noise Models," Trends in Renewable Energy, vol. 2, no. 2, pp. 61-82, 
Jan. $2016 . \quad$ [Online]. Available:

http://futureenergysp.com/index.php/tre/article/view/23

[12] Homeplug, AV2Whitepaper, 2011, [Online]. Available: http://www.homeplug.org/techresources/resources/

[13] A. G. Lazaropoulos, "Factors Influencing Broadband Transmission Characteristics of Underground Low-Voltage Distribution Networks," IET Commun., vol. 6, no. 17, pp. 2886-2893, Nov. 2012.

[14] A. G. Lazaropoulos, "Towards broadband over power lines systems integration: Transmission characteristics of underground low-voltage distribution power lines," Progress in Electromagnetics Research B, 39, pp. 89-114, 2012. [Online]. Available: http://www.jpier.org/PIERB/pierb39/05.12012409.pdf

[15] A. G. Lazaropoulos, "Broadband transmission and statistical performance properties of overhead high-voltage transmission networks,"Hindawi Journal of Computer Networks and Commun., 2012, article ID 875632, 2012. [Online]. Available: http://www.hindawi.com/journals/jenc/aip/875632/

[16] A. G. Lazaropoulos, "Towards modal integration of overhead and underground low-voltage and medium-voltage power line communication channels in the smart grid landscape: model expansion, broadband signal transmission characteristics, and statistical performance metrics (Invited Paper)," ISRN Signal Processing, vol. 2012, Article ID 121628, 17 pages, 2012. [Online]. Available: http://www.isrn.com/journals/sp/aip/121628/

[17] A. G. Lazaropoulos, "Review and Progress towards the Common Broadband Management of High-Voltage Transmission Grids: Model Expansion and Comparative Modal Analysis," ISRN Electronics, vol. 2012, Article ID 935286, pp. $1-18,2012 . \quad$ [Online]. Available: http://www.hindawi.com/isrn/electronics/2012/935286/

[18] A. G. Lazaropoulos, "Review and Progress towards the Capacity Boost of Overhead and Underground Medium-Voltage and Low-Voltage Broadband over Power Lines Networks: Cooperative Communications through Two- and ThreeHop Repeater Systems," ISRN Electronics, vol. 2013, Article ID 472190, pp. 1-19, 2013. [Online]. Available: http://www.hindawi.com/isrn/electronics/aip/472190/

[19] A. G. Lazaropoulos, "Green Overhead and Underground Multiple-Input MultipleOutput Medium Voltage Broadband over Power Lines Networks: EnergyEfficient Power Control," Springer Journal of Global Optimization, vol. 2012 / Print ISSN 0925-5001, pp. 1-28, Oct. 2012.

[20] P. Amirshahi and M. Kavehrad, "High-frequency characteristics of overhead multiconductor power lines for broadband communications," IEEE J. Sel. Areas Commun., vol. 24, no. 7, pp. 1292-1303, Jul. 2006.

[21] T. Sartenaer, "Multiuser communications over frequency selective wired channels and applications to the powerline access network" Ph.D. dissertation, Univ. Catholique Louvain, Louvain-la-Neuve, Belgium, Sep. 2004. [Online\} Available: https://dial.uclouvain.be/pr/boreal/en/object/boreal\%3A5010/datastream/PDF 12/ view

[22] T. Calliacoudas and F. Issa, "“Multiconductor transmission lines and cables solver," An efficient simulation tool for plc channel networks development," presented at the IEEE Int. Conf. Power Line Communications and Its Applications, Athens, Greece, Mar. 2002. 
[23] A. G. Lazaropoulos, "Measurement Differences, Faults and Instabilities in Intelligent Energy Systems - Part 1: Identification of Overhead High-Voltage Broadband over Power Lines Network Topologies by Applying Topology Identification Methodology (TIM)," Trends in Renewable Energy, vol. 2, no. 3, pp. $85-112$, Oct. 2016.

[24] A. G. Lazaropoulos, "Measurement Differences, Faults and Instabilities in Intelligent Energy Systems - Part 2: Fault and Instability Prediction in Overhead High-Voltage Broadband over Power Lines Networks by Applying Fault and Instability Identification Methodology (FIIM)," Trends in Renewable Energy, vol. 2, no. 3, pp. 113 - 142, Oct. 2016. [Online]. Available: http://futureenergysp.com/index.php/tre/article/view/27/33

[25] A. G. Lazaropoulos, "Power Systems Stability through Piecewise Monotonic Data Approximations - Part 1: Comparative Benchmarking of L1PMA, L2WPMA and L2CXCV in Overhead Medium-Voltage Broadband over Power Lines Networks," Trends in Renewable Energy, vol. 3, no. 1, pp. 2 - 32, Jan. 2017. [Online]. Available: http://futureenergysp.com/index.php/tre/article/view/29/34

[26] A. G. Lazaropoulos, "Power Systems Stability through Piecewise Monotonic Data Approximations - Part 2: Adaptive Number of Monotonic Sections and Performance of L1PMA, L2WPMA and L2CXCV in Overhead Medium-Voltage Broadband over Power Lines Networks," Trends in Renewable Energy, vol. 3, no. 1, pp. $33-60$, Jan. 2017. [Online]. Available: http://futureenergysp.com/index.php/tre/article/view/30/35

[27] A. G. Lazaropoulos, "Improvement of Power Systems Stability by Applying Topology Identification Methodology (TIM) and Fault and Instability Identification Methodology (FIIM) - Study of the Overhead Medium-Voltage Broadband over Power Lines (OV MV BPL) Networks Case," Trends in Renewable Energy, vol. 3, no. 2, pp. 102 - 128, Apr. 2017. [Online]. Available: http://futureenergysp.com/index.php/tre/article/view/34/pdf

[28] P. Amirshahi, "Broadband access and home networking through powerline networks" Ph.D. dissertation, Pennsylvania State Univ., University Park, PA, May $2006 . \quad$ [Online]. Available: http://etda.libraries.psu.edu/theses/approved/WorldWideIndex/ETD1205/index.html

[29] M. D'Amore and M. S. Sarto, “Simulation models of a dissipative transmission line above a lossy ground for a wide-frequency range-Part I: Single conductor configuration," IEEE Trans. Electromagn. Compat., vol. 38, no. 2, pp. 127-138, May 1996.

[30] M. D'Amore and M. S. Sarto, "Simulation models of a dissipative transmission line above a lossy ground for a wide-frequency range-Part II: Multi-conductor configuration," IEEE Trans. Electromagn. Compat., vol. 38, no. 2, pp. 139-149, May 1996.

[31] A. Milioudis, G. T. Andreou, and D. P. Labridis, "Detection and location of high impedance faults in multiconductor overhead distribution lines using power line communication devices," IEEE Trans. on Smart Grid, vol. 6, no. 2, pp. 894-902, 2015.

[32] A. G. Lazaropoulos, "Designing Broadband over Power Lines Networks Using the Techno-Economic Pedagogical (TEP) Method - Part I: Overhead High Voltage Networks and Their Capacity Characteristics," Trends in Renewable 
Energy, vol. 1, no. 1, pp. 16-42, Mar. 2015. [Online]. Available: http://futureenergysp.com/index.php/tre/article/view/2

[33] A. G. Lazaropoulos, "Designing Broadband over Power Lines Networks Using the Techno-Economic Pedagogical (TEP) Method - Part II: Overhead LowVoltage and Medium-Voltage Channels and Their Modal Transmission Characteristics," Trends in Renewable Energy, vol. 1, no. 2, pp. 59-86, Jun. 2015. [Online]. Available: http://futureenergysp.com/index.php/tre/article/view/6/16

[34] T. Sartenaer and P. Delogne, "Deterministic modelling of the (Shielded) outdoor powerline channel based on the multiconductor transmission line equations," IEEE J. Sel. Areas Commun., vol. 24, no. 7, pp. 1277-1291, Jul. 2006.

[35] A. G. Lazaropoulos, "Policies for Carbon Energy Footprint Reduction of Overhead Multiple-Input Multiple-Output High Voltage Broadband over Power Lines Networks," Trends in Renewable Energy, vol. 1, no. 2, pp. 87-118, Jun. 2015. [Online].

Available: http://futureenergysp.com/index.php/tre/article/view/11/17

[36] A. G. Lazaropoulos, "Wireless Sensor Network Design for Transmission Line Monitoring, Metering and Controlling: Introducing Broadband over PowerLinesenhanced Network Model (BPLeNM)," ISRN Power Engineering, vol. 2014, Article ID 894628, 22 pages, 2014. doi:10.1155/2014/894628. [Online]. Available: http://www.hindawi.com/journals/isrn.power.engineering/2014/894628/

[37] A. G. Lazaropoulos, "Wireless Sensors and Broadband over PowerLines Networks: The Performance of Broadband over PowerLines-enhanced Network Model (BPLeNM) (Invited Paper)," ICAS Publishing Group Transaction on IoT and Cloud Computing, vol. 2, no. 3, pp. 1-35, 2014. [Online]. Available: http://citeseerx.ist.psu.edu/viewdoc/download;jsessionid=741EE7C15693046FFF F5E9749149F579?doi=10.1.1.679.8217\&rep=rep1\&type $=$ pdf

[38] A. G. Lazaropoulos, "The Impact of Noise Models on Capacity Performance of Distribution Broadband over Power Lines (BPL) Networks," Hindawi Computer Networks and Communications, vol. 2016, Article ID 5680850, 14 pages, 2016. doi:10.1155/2016/5680850. [Online].
http://www.hindawi.com/journals/icnc/2016/5680850/

[39] A. N. Milioudis, G. T. Andreou, and D. P. Labridis, "Enhanced Protection Scheme for Smart Grids Using Power Line Communications Techniques-Part II: Location of High Impedance Fault Position," IEEE Trans. on Smart Grid, no. 3, vol. 4, pp. 1631-1640, 2012.

[40] A. Milioudis, G. Andreou, and D. Labridis, "High impedance fault detection using power line communication techniques," in Proc. $201045^{\text {th }}$ Int. Univ. Power Eng. Conf. (UPEC), pp. 1-6, Cardiff, U.K., 2010.

[41] A. Milioudis, G. Andreou, and D. Labridis, "High impedance fault evaluation using narrowband power line communication techniques," in Proc. 2011 IEEE Trondheim PowerTech, Trondheim, Norway, pp. 1-6.

[42] A. Milioudis, G. Andreou, and D. Labridis, "Enhanced protection scheme for smart grids using power line communications techniques-Part I: Detection of high impedance fault occurrence," IEEE Trans. Smart Grid, vol. 3, no. 4, pp. 1621-1630, Dec. 2012.

[43] A. G. Lazaropoulos, "Deployment Concepts for Overhead High Voltage Broadband over Power Lines Connections with Two-Hop Repeater System: Capacity Countermeasures against Aggravated Topologies and High Noise 
Environments," Progress in Electromagnetics Research B, vol. 44, pp. 283-307, 2012. [Online]. Available: http://www.jpier.org/PIERB/pierb44/13.12081104.pdf

[44] S. Liu, F. Yang, and J. Song, "An Optimal Interleaving Scheme with Maximum Time-Frequency Diversity for PLC Systems," IEEE Trans. on Power Del., vol. 31, no. 3, pp. 1007-1014, 2016.

[45] G. Prasad, L. Lampe, and S. Shekhar, "In-Band Full Duplex Broadband Power Line Communications," IEEE Trans. on Commun., vol. 64, no. 9, pp. 3915-3931, 2016.

[46] A. G. Lazaropoulos, "Main Line Fault Localization Methodology in Smart Grid Part 3: Main Line Fault Localization Methodology (MLFLM)," Trends in Renewable Energy, vol. 3, no. 3, pp. 62-81, 2017.

[47] A. G. Lazaropoulos, "Main Line Fault Localization Methodology in Smart Grid Part 2: Extended TM2 Method, Measurement Differences and L1 Piecewise Monotonic Data Approximation for the Overhead Medium-Voltage Broadband over Power Lines Networks Case," Trends in Renewable Energy, vol. 3, no. 3, pp. 26-61, 2017.

Article copyright: (C) 2017 Athanasios G. Lazaropoulos. This is an open access article distributed under the terms of the Creative Commons Attribution 4.0 International License, which permits unrestricted use and distribution provided the original author and source are credited. 